\title{
Cultivating the Science Internet Audience
}

Impact of Brand and Domain on Source

Credibility for Science Information

\author{
DEBBIE TREISE \\ KIM WALSH-CHILDERS \\ MICHAEL F. WEIGOLD \\ MEREDITH FRIEDMAN
}

University of Florida

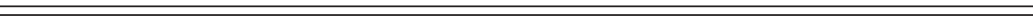

This two-part study examines factors that can influence the perceived credibility of a science Web site. In the first part, respondents answered questions about their perceptions of science sites with the .com and gov domain. Findings suggest that sites with a gov domain are perceived to be more credible. Part 2 is an experiment in which respondents read a short science article from a site. Participants were randomly assigned to experimental conditions in which they were led to believe the site was from either a high-or moderate-credibility source and from a domain that contained either a gov or .com extension. Ratings of the author, source, and the article were systematically influenced by the manipulations. Implications for science Web sites are presented in the discussion.
\end{abstract}

Keywords: science communication; Internet credibility; domain credibility

The popularity of the Internet has challenged many core assumptions about relationships between sources, mass media, and audiences in many areas of

Authors'Note: The authors would like to thank Ron Koczor and Science@NASA, Marshall Space Flight Center for their support and cooperation in this project. Address correspondence to Debbie Treise, University of Florida, College of Journalism and Communications, 2084 Weimer Hall, Gainesville, FL 32611; phone: 352-392-9755; fax: 352-846-3015; e-mail: dtreise@ jou.ufl.edu.

Science Communication, Vol. 24 No. 3, March 2003 309-332

DOI: $10.1177 / 1075547002250298$

(C) 2003 Sage Publications 
communication but perhaps most dramatically in the area of science communication (Weigold 2002). Traditionally, the cost and complexity of producing any mass-mediated product limited the numbers of information-producing people and organizations an audience member would be exposed to, thereby not only limiting the range of choices consumers have in terms of information providers but also increasing the likelihood that those providing information would have a substantial investment in ensuring its accuracy. However, because of the ease and cost effectiveness with which information can be posted on the Web, the number of sites has exploded over the past ten years. One well-known source of science information recently suggested that there are now 550 billion unique documents on the Web, with approximately 7 million pages being added daily (Popular Science 2001).

For consumers of science information, the Web presents both opportunities and challenges. By entering terms into a search engine, consumers may quickly discover that there are hundreds or even thousands of sites that offer science information with the click of a mouse. Knowing which of the many sites available contains information high in quality, currency, and accuracy is a much greater challenge. Because anyone can post information on the Web, finding science information that is useful, accurate, and current may be difficult (Hartz and Chappell 1997). The explosion of information about science on the Web may pose a challenge for sites as well. It may be difficult for quality sites to distinguish themselves from competitors offering information of lower quality or veracity.

This study focuses on a common currency for sources and audiences of science information: credibility. The primary purpose of this study is to determine the effects of a prestigious versus generic source and a .gov versus .com domain on perceptions of the credibility of an online source and a science story. The credibility of online science is largely unexplored, as opposed to other areas, such as health information, that have received much attention (Weigold 2002). Although nongovernment organizations do not have the option of using the gov domain, governmental organizations do have the option of changing their Web addresses to use a .com extension. Such a change might be attractive to some government-sponsored science agencies because many Web users may not be familiar with the gov domain, may believe all Web sites have a .com domain, or may not realize that agencies such as NASA, the Centers for Disease Control and Prevention, or the National Institutes of Health are governmental, not commercial, agencies.

Although source credibility is only one dimension that may attract people to a site (others may include ease of use, attractiveness, organization, writing quality, etc.), it may be an important one because audiences use source credibility as a cue to the quality and veracity of messages. Messages from high- as 
compared to low-credibility sources are more believable and persuasive because high-credibility sources are considered to be higher in expertise and trustworthiness (Sternthal, Phillips, and Dholakia 1978).

\section{Credibility and Audience Responses}

The two most consistently documented dimensions of the credibility of an information source (whether interpersonal or mediated, such as a Web site) are trustworthiness and expertise (Sternthal, Phillips, and Dholakia 1978). Expertise generally refers to the audience's perceptions of the source's capability for providing correct information. Trustworthiness, on the other hand, refers to the audience's perceptions of the likelihood that the source is providing information the source believes to be true or valid. Prior research has demonstrated, not surprisingly, that audiences are more likely to accept or be persuaded by sources perceived to have greater expertise and to be more trustworthy (Marquart, O'Keefe, and Gunther 1995; Sternthal, Phillips, and Dholakia 1978; Wiener and Mowen 1986).

Audiences may employ different criteria for judging the credibility of information depending on the medium in which it is presented (Newhagen and Nass 1989), which suggests that the criteria by which audiences evaluate Web site information may differ from those used for more traditional media. What are the criteria that Web audiences use to evaluate the credibility of Web sites? The literature in this area so far can be divided into two categories: discussions of the criteria various individuals and organizations recommend for judging the credibility of online information and research focusing on audiences' perceptions of the credibility of online information, sometimes in comparison to their perceptions of information provided by traditional news channels such as newspapers. As an example of the first category, the Health on the Net Foundation (2001) urged users of online information to verify that the Web sites they are using clearly display the credentials of authors who have written the sites' information, the date the pages were last updated, the Web site's policy on confidentiality of data provided by users, references for the sources of data used in the information, and the Web site's sources of funding and advertising policies. Hawkins (1999) rank ordered the credibility criteria most frequently mentioned in fourteen sites on information quality listed in the Evaluation of Information Resources page of the WWW Virtual Library. He reported that the most frequently mentioned questions dealt with the currency of the site's information, the purpose or audience for the site, apparent bias or objectivity of the information, and questions about the author or publisher of the information, including whether the author's name 
appears on the site, whether the author is well known and well qualified, and whether the publisher represents a reputable organization.

While these criteria may reflect standards audiences should use to judge Web-based information credibility, there is relatively little research to date demonstrating that in fact people do use these criteria in evaluating online information or source credibility. However, researchers have begun to analyze at least the extent to which audiences judge online information as credible. For instance, a survey sponsored by the Online News Association (Finberg, Stone, and Lynch 2001) revealed that those who use online news sites rate them as being about as credible as traditional news sources; cable television news programs and national newspapers were rated as most credible, but the third-ranked news source was cable TV-related Web sites (i.e., CNN.com). However, the study also showed that the online reading public is less certain or more neutral about the credibility of online news sources, while these people are much more likely to have strong opinions about the credibility of traditional news sources (Finberg, Stone, and Lynch 2001). Johnson and Kaye (1998) used an online survey to investigate how politically interested Web users compared the credibility of online political information versus political information from traditional print sources. Their findings showed that the survey respondents generally rated both online and traditional sources of political information as only "somewhat" credible. However, the respondents rated online newspapers and online candidate literature as significantly more credible than print newspapers and candidate literature. Reliance on a medium - either print or online - for political information was positively and significantly associated with credibility ratings for that particular medium; for example, those who relied on print newspapers for political information rated them as more credible compared to the print newspaper ratings of those who did not rely on print newspapers.

Similarly, Flanagin and Metzger (2000) found that their survey respondents viewed online information to be as credible as that found in television, radio, and magazines but less credible than information found in newspapers. Sundar (1999) investigated the criteria respondents use in rating print and online news stories by having college students read print or online stories and then rate them using twenty-one adjectives, including accurate, believable, interesting, relevant, sensationalistic, well written, and others. Factor analysis of the ratings of the stories revealed four factors for both print and online news stories, which Sundar labeled Credibility, Liking, Quality, and Representativeness. The recent survey conducted by the Online News Association (Finberg, Stone, and Lynch 2001) concluded that the term credibility is slippery indeed and that the public and journalists hold highly divergent views of it. However, the study also concluded that online news outlets are deemed at 
least as credible as other traditional media outlets, but "online news credibility is not a top-of-mind issue for the online public" as yet-they just "haven't made up their minds" (Lasica 2002).

These findings suggest, first, that perceptions of online science story credibility may vary in part according to the readers' reliance on or frequency of use of online science Web sites. Those who use science Web sites more frequently, regardless of the type of Web site, might be expected to rate online science stories as more credible than would readers who visit science Web sites only infrequently or who prefer more traditional sources of science information. Second, the findings suggest that the same factors that influence perceptions of a newspaper story's credibility may affect perceptions of stories published online; for instance, if judgments about a newspaper's reputation for accuracy and objectivity influence story credibility perceptions, then readers' beliefs about the probable accuracy and lack of bias of a Web site should influence online story credibility perceptions.

We were able to locate only one study specifically focusing on use of science Web sites. Eveland and Dunwoody's (1998) investigation of individuals' use of The Why Files, a science Web site produced by the Graduate School at the University of Wisconsin-Madison, analyzed the patterns of usage among repeat visitors to The Why Files site. Findings revealed that women were more interested than men in information about medical discoveries and environmental issues, but men were significantly more interested in new technology information. Older respondents, not surprisingly, also were more interested in medical discoveries than were younger respondents. The researchers did not collect data related to respondents' evaluations of the Web site's credibility.

There has been at least one study exploring scholars' use of Web sites related to their discipline, focusing on how users judged the authority and quality of the information they found. Rieh and Belkin (1998) conducted indepth interviews with fourteen faculty members and doctoral students in communication, library and information studies, computer science, education, psychology, social psychology, and organizational psychology. They found that the scholars' judgments about online information recognized source credibility at two levels-institutional and individual. Institutional authority related to the domain of the Web site, the type of institution, and the institution's reputation.

Rieh and Belkin's (1998) respondents generally perceived .edu and .gov sites as providing better quality information than .com sites. Respondents also based their judgments of information quality in part on the type of institution. For instance, the respondents noted that universities, museums, libraries, and professional associations all were likely to provide credible 
information. In addition, the name of the particular institutions (i.e., Stanford, MIT, Microsoft, IBM) was important in some respondents' evaluations of information quality. These findings suggest that science story readers would give higher credibility ratings to stories from a .gov Web site than to a .com Web site and, furthermore, that having a well-known agency name such as NASA as part of the Web site address should significantly increase perceived story credibility.

At the individual level, respondents made judgments based on the names of the authors or creators of Web pages. Some respondents noted that authors who identify themselves and include their addresses and/or other contact information and the name of their institutions enhanced the credibility of the Web site. Author affiliations, such as the person's occupation or the institution for which he or she worked, influenced respondents' perceptions of information credibility. And as with the institutional level, some respondents noted that recognizing an author's individual name and reputation affected their evaluations of the information (Rieh and Belkin 1998). Their analysis, unfortunately, did not include a comparison of the importance of individuallevel versus institutional-level factors or a direct comparison of the credibility of various domains; however, the fact that an individual author's credibility was influenced in some cases by the reputation of the institution for which he or she worked suggests that institutional-level factors may dominate. In addition, readers of any given story are far more likely to be familiar with the reputation of an agency such as NASA than to know the reputation of the story's author.

Another finding of the study that is highly pertinent to our research was that the scholars' commitment to evaluating information quality varied depending on the purpose for which they were using the information. If the consequences of using bad information were deemed serious, the scholars reported that they would be more cautious about checking the quality of the online information. Similarly, if the respondent was going to take action based on the information - and particularly if that action would require spending money or other substantial resources - then evaluating information quality was more important. Finally, the importance of ensuring the quality of the information increased as the goal for information seeking became more focused (Rieh and Belkin 1998).

Flanagin and Metzger (2000) reported somewhat similar findings in their research on perceptions of online information credibility. Their data revealed that the credibility of various media varied depending on the type of information sought, with news, reference (factual nonnews), and entertainment information ${ }^{1}$ judged more credible than commercial information regardless of which medium carried the information. For online information, news, 
reference, and entertainment information was perceived to be more credible than commercial information, and reference information was judged more credible than entertainment information. In addition, the researchers found that users expended more effort toward verifying reference information than they did commercial or entertainment information. They made a greater attempt to verify news information than they did entertainment information, but news and commercial information was verified no differently.

\section{Uses and Gratifications}

These findings (Flanagin and Metzger 2000; Rieh and Belkin 1998) also suggest another theoretical perspective that may need to be considered in making predictions about how users will judge the credibility of online science information. Uses and gratifications theory acknowledges that audiences do not simply expose themselves to information randomly; rather, they actively choose different media channels and types of information purposively, depending on their particular goals and their expectations about how well the media channels and information types will meet those goals. Katz, Blumler, and Gurevitch (1974) summarized the uses and gratifications approach as

(1) the social and psychological originals of (2) needs, which generate (3) expectations of (4) mass media or other sources, which lead to (5) differential patterns of media exposure (or engagement in other activities), resulting in (6) need gratifications and (7) other consequences, perhaps mostly unintended ones. (P. 20)

Blumler (1979) argued that the research indicates that there are three major types of uses for which people turn to the media. Cognitive uses related to individuals' use of the media to acquire information or to better understand something. Diversion or escape uses were those related to getting away from one's personal problems and concerns and seeking emotional release. Personal identity concerns related to use of the media to validate or reinforce one's values or to achieve better self-understanding.

There has been at least one study of the gratifications sought by Internet users. Papacharissi and Rubin (2000) found that among undergraduate college students, five motives drove Internet use: interpersonal utility (e.g., to belong to a group, to participate in discussions, to express oneself freely), passing the time, information seeking, convenience, and entertainment. Instrumental uses of the Internet, such as seeking information, were 
associated with students who were economically secure and comfortable in interpersonal interactions.

\section{Involvement}

Researchers have found that source credibility interacts with characteristics of the individual receivers, including individual versus external locus of control, authoritarianism, and involvement in the issue discussed in the source's communication (Sternthal, Phillips, and Dholakia 1978). Perhaps most important for this study, researchers have found that involvement with an issue mediates the effects of source credibility. Johnson and Scileppi (1969) and Rhine and Severance (1970) found that when audience members' involvement with an issue was low, a highly credible source was more persuasive than a low-credibility source. However, when audience members were highly involved in the issue, credibility did not significantly affect persuasiveness. Other researchers (i.e., Gunther 1988) have found curvilinear relationships between source credibility and believability, depending on audience members' involvement with a particular issue. Thus, the research suggests that the relationships between science Web site characteristics and the credibility of stories found on those Web sites may differ among readers with greater or lesser involvement in science.

In general then, audiences who are highly involved with a message (who possess sufficient knowledge to make sense of the message and sufficient motivation to process a message carefully) may be most likely to evaluate the quality of the arguments contained in a message itself. The highly involved audience member is likely to think about the message, evaluate the merits of the arguments contained therein, and form opinions about the message based on the quality of the arguments (strong arguments lead to more favorable opinions; weak arguments lead to less favorable opinions). A less involved audience member, particularly one who lacks the ability to process a message (for example, because the message is complex or because it deals with topics for which the audience member possesses little knowledge), forms opinions about the message based on factors other than the arguments contained therein. Research suggests that such a person is more likely to form an opinion about the message by using cues that "surround" the message, such as the credibility of the source.

This reliance of low-involvement audience members on message cues is especially important in science communication because Miller (1998) estimated that only 5 percent of the U.S. population and only 12 percent of adults 
with college degrees are involved enough with science to be considered scientifically "literate." While the definition and measurement of science literacy are hotly debated, it "commonly implies as appreciation of the nature, aims and general limitations of science, coupled with some understanding of the more important scientific ideas" (Jenkins 1994, 5345). Defining characteristics of the scientifically literate are their motivation and ability to seek new information about science (Sapp 1992). However, little is known about how consumers of these Web-based science stories use and evaluate the credibility of the information they are uncovering.

Summary. Previous research had not specifically linked the traditional source credibility literature with the small but growing body of research on audience perceptions of the credibility of information available online. However, the research does suggest that readers' perceptions of the credibility of online science stories may be influenced by the readers' involvement with science and perhaps by their motivations for reading online science stories. In addition, both surveys of online information users and the recommendations various organizations have made for evaluating online information suggest that readers will perceive a story from a .gov Web site as more credible than one from a .com Web site. Evidence also suggests that a well-known institution name, such as NASA, is likely to increase readers' perceptions of story credibility. Therefore, we tested the following hypotheses:

Hypothesis 1: Credibility scores for online science stories from a .gov Web site will be higher than those for the same stories from a .com Web site.

Hypothesis 2: The effect of the domain (.gov versus .com) will be greater for those who are low as compared to high in science involvement.

Hypothesis 3: Credibility scores for online science stories from a prestigious source will be higher than those for the same stories from a generic source.

Hypothesis 4: The effect of the source on credibility ratings will be greater for respondents who are low as compared to high in science involvement.

We also wished to explore the relationships between self-reported use of the Web for obtaining science information, motivations (uses and gratifications) for science information, and science involvement. The literature in this area does not suggest any clear directions for these relationships. Hence, we investigated the following research question:

Research Question: What relationships characterize use of the Web for obtaining science information, motivations for science information, and science involvement? 


\section{Method}

\section{Participants}

There were 497 male and female undergraduate students recruited from large introductory science, engineering, and mass communication courses at a large southeastern university. College students were used as the sample population because we believed they would represent a larger proportion of science literates than the general populace. In most cases, the students were awarded extra credit for their participation in the study. In the remaining cases, instructors asked for participants on a volunteer basis only.

\section{Procedure}

The study encompassed two data collection methods administered sequentially. Although identical participants were involved in both methods, it simplifies the presentation to focus on each collection method separately. Part 1 was used primarily to test hypothesis 1 concerning domain credibility (a .gov domain will be higher in credibility than a .com domain) and hypothesis 2 concerning the impact of science involvement on credibility (the credibility superiority of the gov domain will be greater for low- as compared to high-science involvement respondents). In addition, part 1 allowed us to address our research question about the relationships between the constructs of science involvement, science information motivation, and the frequency of science Web site use.

The science background of respondents was measured several ways. First, respondents were asked to indicate the number of college-level courses they had taken in each of the following areas: biology, chemistry, astronomy, earth science, physics, and other sciences. Second, a measure of self-perceived literacy was obtained by asking respondents to compare themselves to other students at their university and "rate your literacy or knowledge about general science issues." Responses were scaled from 1 (not at all literate) to 7 (very literate). Similar language and the same scale were used to measure self-perceived literacy for "the area of science (biology, chemistry, physics, astronomy, etc.) that you are most interested in."

As a measure of science Web site use, respondents were asked about the frequency with which they used the Web to find science information (almost every day, several times a week, once or twice a week, once or twice a month, or fewer than twelve times a year). Participants also were queried about their motives for obtaining that information (i.e., to obtain knowledge useful for school- or work-related projects, to satisfy curiosity, to pass time, to monitor 
new developments in science or a field of science, for entertainment, to develop a deeper understanding of science or a field of science, to obtain knowledge useful for conversations with others, to obtain knowledge useful for my current/future career, to verify facts or information I have learned about elsewhere). Each motive was rated on scales that ranged from 1 (very important) to 7 (very unimportant). We queried respondents about the importance of three Web site attributes for using the site. These attributes were the credibility of the sponsor, attractiveness, and completeness of information. For each, respondents used 5-point Likert-type scales in response to statements that read, "The ___ of a site is an important consideration in whether I will visit the site to obtain science information."

Two measures were used to assess domain credibility. First, respondents were asked to imagine

you were searching the Web for information about a science issue. If you got conflicting or different reports of the same story from a .com, a .org, a .edu, and a .gov site, which of the four versions would you be most inclined to believe?

Second, respondents were asked to imagine visiting two science Web sites, the first with a .com extension and the second with a .gov extension. For each domain, respondents provided ratings on a set of 9-point bipolar rating scales as to their expectations for such a site. The scales were Expert-Not Expert, Experience-Not Experience, Trained-Untrained, Trustworthy-Not Trustworthy, Moral-Immoral, Good-Bad, Reliable-Unreliable, InformedUninformed, Qualified-Unqualified, Intelligent-Unintelligent, ValuableWorthless, Honest-Dishonest, Friendly-Unfriendly, Pleasant-Unpleasant, Unselfish-Selfish, and Valuable-Useless.

Part 2 was a randomized experiment that could test all four hypotheses, including replications of the two tested in part 1, as well as hypothesis 3 (a story attributed to NASA's Web site would be perceived as more credible than one attributed to a generic science site) and hypothesis 4 (the credibility advantage for a story associated with NASA versus a generic site would be more pronounced for low- as compared to high-involvement respondents). In replicating the first two hypotheses, part 2 addressed an important deficiency of part 1 because it required respondents to rate information attributed to a specific science site and not an imagined site. Participants were asked to read an actual science story (obtained from NASA's Web site) that was randomly attributed either to NASA or to a generic science site (Spacescience). Students were given the hard copies of the articles; researchers did not call attention to the URLs. Crossed with this manipulation, participants were randomly led to believe that the story's domain was either .com (indicating a 
commercial site) or .gov (indicating a government site). To increase generalizability, participants read one of five short stories from different fields of science. Information about the source and domain was presented using directions that read, "On the following page is an article taken from the Web. The Web site is Please read the article carefully in order to answer questions that appear later on." The article appeared on the next page, where the Web address was repeated again.

The final design was thus a 2 (domain: .gov versus .com) $\times 2$ (highcredibility source [NASA] versus medium-credibility source [Spacescience]) $\times 5$ (story: astronomy, biology, earth science, physiology, physics) factorial. After reading one of the stories, participants completed ratings of the source (expert-not expert, experienced-not experienced, trained-untrained, trustworthy-not trustworthy, moral-immoral, good-bad, reliable-unreliable, informed-uninformed, qualified-unqualified, intelligentunintelligent, valuable-worthless, expert-inexpert, honest-dishonest, friendly-unfriendly, pleasant-unpleasant, unselfish-selfish), author (fair-unfair, biased-unbiased, accurate-inaccurate, watches after reader's interests-does not watch after reader's interests, separates fact and opinion-does not separate fact and opinion, concerned about the public interest-concerned about making profits, factual-opinionated, well trained-poorly trained, and concerned about what the audience thinks-is not concerned what the audience thinks), and article (useful-useless, valuable-not valuable, persuasive-not persuasive, complete-incomplete, easy to understand-difficult to understand, relevant-irrelevant). All measures were obtained on 9-point bipolar rating scales.

\section{Findings}

\section{Part 1: Motives for Using Science Web Sites and Ratings of Generic Domains}

Preliminary analyses. The Science Involvement Scale was created by summing measures of (1) the number of college-level courses students had taken in biology, chemistry, astronomy, earth science, physics, and other sciences; (2) self-rated literacy about general science issues; (3) self-rated literacy about "the area of science that you are most interested in"; (4) the importance of staying informed about general science issues; and (5) the importance of staying informed about issues in the area of science for which the student indicated greatest interest. The resulting scale $(M=21, S D=6.5)$ possessed good reliability (Cronbach's alpha $=.70$ ). 
Background characteristics of the sample are presented in Table 1. The table shows that the median number of science courses for our sample was 2.5. A background of coursework in biology and chemistry tended to be more common than a background in astronomy, earth science, physics, or other science courses. Respondents also indicated the frequency with which they use the Web to obtain information about science. Responses included never (0), fewer than twelve times a year (1), once or twice a month (2), once or twice a week (3), several times a week (4), and almost every day (5). The largest percentage (35 percent) indicated that they use the Web for science information fewer than twelve times a year. About 22 percent never use the Web for science information. An additional 22 percent use the Web once or twice a month, 13 percent use it once or twice a week, 4 percent use it several times a week, and 3 percent use it almost every day.

To better understand the structure of motives for visiting a science Web site, we conducted a factor analysis on the motive items. ${ }^{2}$ The analysis resulted in two factors that together accounted for 63 percent of the variance. Using these criteria, the resulting factors were labeled Personal Motives (for entertainment, to pass time, to satisfy curiosity, to monitor new developments, to learn information useful for conversation, and to develop a deeper understanding) and Functional Motives (knowledge for school and knowledge for my career). One item, "to verify facts learned elsewhere," loaded highly on both factors and was treated separately.

Primary analyses. The measure of frequency for using the Web for science information was correlated with the Science Motive Scales and with science involvement. All of these correlations proved strongly positive and significant (see Table 1). Motives related to personal usage, functional usage, and verifying facts were positively associated with science involvement. In addition, using the Web to obtain information about science was strongly and positively related to involvement and all three motives. These results confirm the importance of the World Wide Web as a source of information useful to individuals who are involved with science. They also suggest that the audience for science information at science Web sites is likely to be more rather than less knowledgeable about science.

The next analysis focused on measures of three site attributes that could be important in determining whether a respondent visits a science Web site. These attributes were sponsor credibility, site attractiveness, and completeness of information. To determine the relative importance of these attributes and to determine whether the attributes differed in importance for those high as compared to low in science involvement, a MANOVA was performed in 
TABLE 1

Correlations of Using the Web to Obtain Science Information with Motives and Involvement

\begin{tabular}{|c|c|c|c|c|c|}
\hline & 1 & 2 & 3 & 4 & 5 \\
\hline 1. Using the Web to obtain science information & & .45 & .45 & .32 & .49 \\
\hline 2. Personal motives & & & .47 & 69 & .45 \\
\hline 3. Functional motives & & & & .45 & .45 \\
\hline 4. Verify facts & & & & & .32 \\
\hline 5. Science involvement & & & & & \\
\hline
\end{tabular}

which attribute was treated as a within-subjects variable and science involvement was treated as a between-subjects variable.

This analysis revealed several important findings. First, the attribute main effect proved highly significant, $F(2,483)=149.72, p<.001$. Examination of the means showed information completeness to be the most important attribute, while site attractiveness was rated as least important (completeness $M=$ 4.24, credibility $M=3.80$, attractiveness $M=3.45$, each measured on 5-point scales). In addition, a significant site attribute by science involvement interaction was found, $F(2,483)=15.33, p<.001$. Means for this interaction are presented in Table 2 . The means suggest that those high versus low in involvement differ in how important they perceive site attractiveness and site completeness to be for visiting a site. Low-involvement respondents as compared to high-involvement respondents assign greater importance to attractiveness, while high-involvement respondents as compared to lowinvolvement respondents assign greater importance to information completeness. These results are consistent with predictions derivable from the elaboration likelihood model because information completeness is related to argument quality (and thus central route processing), while attractiveness often serves as a peripheral cue.

Science site attributes also were examined as a function of the motives scales by computing correlations. Results suggested that both personal motives $(r=.12)$ and verifying facts $(r=.14)$ were positively related to the importance of sponsor credibility. In addition, personal motives $(r=.20)$, functional motives $(r=.19)$, and verifying facts $(r=.20)$ were related to the importance of information completeness. None of the motives were correlated with the importance of site attractiveness.

Domain credibility. The credibility of different domains was explored two different ways. First, respondents were asked to imagine that in searching the Web for information about a science issue, they received conflicting reports 
TABLE 2

Science Web Site Attribute Importance by Science Involvement

\begin{tabular}{lcc}
\hline \hline Source Attribute & Low Involvement & High Involvement \\
\hline Sponsor credibility & $3.74_{\mathrm{a}}$ & $3.86_{\mathrm{a}}$ \\
Attractiveness & $3.59_{\mathrm{a}}$ & $3.29_{\mathrm{b}}$ \\
Completeness & $4.13_{\mathrm{b}}$ & $4.38_{\mathrm{c}}$ \\
\hline
\end{tabular}

NOTE: Row and column means with different subscripts differ significantly $(p<.05)$.

of the same story from a .com, .org, .edu, and .gov site. Participants were instructed to indicate which of the four versions they would be most inclined to believe. A significant chi-square, $\chi^{2}(4,521.29), p<.001$, indicated that responses to this question were not equally distributed. A majority of individuals selected the .edu site (57 percent). Following, in order of popularity, were the .gov (26 percent), .org (10 percent), and .com (6 percent) sites. A cross-tabulation analysis of this measure with science involvement suggested no relationship between the two variables.

Respondents also rated expectations regarding science Web sites, first for one with a .com extension and next for one with a .gov extension. The domain credibility ratings were separately factor analyzed for .gov and .com, with each analysis confirming the presence of two factors (accounting for 76 percent of the variance for gov sites and 65 percent of the variance for .com sites). Each domain evinced one factor that could be labeled Expertise (informed, reliable, qualified, expert, valuable, useful, experienced, and intelligent) and a second factor that could be labeled Attractiveness (friendly, pleasant, unselfish, and good).

These four measures (.gov expertise, .gov attractiveness, .com expertise, and .com attractiveness) were then subjected to a MANOVA in which science involvement was treated as a between-subjects variable and domain and credibility dimensions were treated as within-subjects variables. Results of the analysis revealed main effects for the domain, $F(1,489)=199.78, p<.001$, and for the credibility factor, $F(1,489)=90.34, p<.001$. As predicted by hypothesis 1 , gov science sites were considered significantly more credible than .com science sites (.gov $M=6.44, \operatorname{com} M=5.43)$, and science sites were rated higher on expertise $(M=6.13)$ than likeability $(M=5.74)$. However, these effects were qualified by two interactions, a two-way interaction of domain by science involvement, $F(1,489)=3.96, p<.05$, and a two-way interaction of domain by credibility factor, $F(1,489)=248.99, p<.001$. Means for the interactions are presented in Tables 3 and 4. 
TABLE 3

Science Web Site Credibility as a Function of Domain and Science Involvement

\begin{tabular}{lll}
\hline \hline Source Attribute & gov & .com \\
\hline Low involvement & $6.43_{\mathrm{a}}$ & $5.28_{\mathrm{b}}$ \\
High involvement & $6.46_{\mathrm{a}}$ & $5.59_{\mathrm{b}}$ \\
\hline
\end{tabular}

NOTE: Row and column means with different subscripts differ significantly $(p<.05)$.

TABLE 4

Science Web Site Credibility as a Function of Domain and Credibility Dimension

\begin{tabular}{lll}
\hline \hline Source Attribute & gov & .com \\
\hline Credibility & $6.98_{\mathrm{a}}$ & $5.28_{\mathrm{c}}$ \\
Likeability & $5.90_{\mathrm{b}}$ & $5.59_{\mathrm{d}}$ \\
\hline
\end{tabular}

NOTE: Row and column means with different subscripts differ significantly $(p<.05)$.

The domain by science involvement interaction bears directly on hypothesis 2 . The pattern of means presented in Table 3 is consistent with the expectation that respondents who were low as compared to high science involved use the domain cue to a somewhat greater extent. However, some caution is warranted because the simple effect of domain is significant for both low- and high-involvement respondents. Furthermore, the simple effect of science involvement in ratings of .com credibility is only marginally significant $(p<$ .10). Table 4 shows means for the domain by credibility dimension interaction and makes clear the perceived importance of domain for credibility ratings: .gov sites are rated as significantly more expert than likable, while .com sites are rated as significantly more likable than expert. Overall, however, .gov science sites are rated as higher in both expertise and likeability than are .com sites.

\section{Discussion: Part 1}

Using two very different measures, we found that respondents judge information at .gov science sites to be more credible than information at .com sites. This offers strong support for hypothesis 1. Qualified support is found for the second hypothesis, which predicted that the effect of domain would be qualified by our respondents' levels of science involvement. The expected involvement by domain interaction did occur, and the pattern of means was 
consistent with our expectations that domain would be a more important cue for low- as compared to high-involvement respondents. But simple effects tests failed to confirm that domain was a significantly stronger cue for lows as compared to highs.

With respect to our research question concerning the relationships between motives for using the Web, science involvement, and frequency of using the Web to obtain science information, our results suggest that each of these variables is strongly and positively related to the others. Those who show high involvement in science (as measured by science background, interest in science, and the importance of staying informed about science) exhibit stronger motives to use the Web for science information and report using the Web to visit science sites more often.

\section{Part 2: Responses to Content from a Science Web Site}

Primary Analyses

Source dimensions. Source dimension items were factor analyzed using principal components analysis with a varimax rotation, resulting in two factors that together accounted for 76 percent of the variance. These factors were labeled Expertise (experienced, qualified, expert, expertise, trained, informed, reliable, intelligent, valuable) and Attractiveness (friendly, pleasant, unselfish, moral). Three highly correlated items (trustworthy, good, and honest) did not load cleanly on either factor. Because the literature on source dimensions has long distinguished trustworthiness and expertise as dimensions of credibility, these three items were summed to create a third scale, which was labeled Trustworthiness (good, honest, trustworthy). The Expertise, Attractiveness, and Trustworthiness Scales possessed alphas of .95, .90, and .87 , respectively.

Science involvement scores were split at the median and used as an independent variable in an analysis of source factors in a multivariate analysis of covariance (MANCOVA). Source dimension (expertise, attractiveness, trustworthiness) was treated as a three-level within-subjects variable, and Science Involvement (high vs. low), Article Source (NASA vs. Spacescience), and Domain (.com vs. .gov) were treated as between-subjects factors. Individual story (five levels) was treated as a covariate. Results showed a significant main effect of the Source factor, $F(2,481)=4.08, p<.02$, and a significant two-way interaction of Source factor by Domain, $F(2,481)=3.34, p<.04$.

The three factors then were analyzed separately using ANCOVA. The Expertise factor showed main effects for Domain, $F(1,483)=4.59, p=.03$, 
and Source, $F(1,483)=7.19, p<.01$. As predicted by hypothesis 3 , respondents attributed greater expertise to the article source when it was identified as NASA $(M=6.73)$ than when it was identified as a generic site $(M=6.39)$. In addition, and consistent with hypothesis 1 , the article source was considered to have greater expertise when associated with a .gov $(M=6.70)$ as compared to a .com domain $(M=6.43)$. However, hypotheses 2 and 4 were not supported. These hypotheses predicted significant two-way interactions for science involvement and source as well as for science involvement and domain. Instead, domain and source were equally important cues for both low- and high-science-involved respondents.

Analysis of the Attractiveness factor revealed only a marginally significant science involvement main effect, low-involvement $M=6.23$; highinvolvement $M=6.47 ; F(1,482)=3.67, p=.06$. No effects were significant for the Trustworthiness factor.

Ratings of the article. Factor analysis of the article characteristics revealed a single factor, and the items were therefore combined into a single Article Quality Scale (useful, valuable, persuasive, complete, easy to understand, relevant). The alpha coefficient for the scale was .86. An ANCOVA (controlling for article) revealed main effects of source, $F(1,480)=6.17, p<.02$, and science involvement, $F(1,480)=4.09, p<.05$. The articles received higher ratings when they were attributed to NASA's Web site $(M=6.54)$ as compared to the generic Web site $(M=6.20)$. In addition, high-involvement $(M=$ $6.50)$ as compared to low-involvement $(M=6.23)$ respondents rated the articles more favorably.

Ratings of the author. Factor analysis of the author ratings revealed one dominant factor and a single item that did not load on the factor. The factor, which we labeled Competence, included the items factual, well trained, accurate, concerned about the public interest, separates fact and opinion, watches out for the reader's interests, fair, and concerned what the audience thinks $(\alpha=.90)$. The lone item that did not load on this factor we labeled objectivity (biased-unbiased). Both the factor and the single item were subjected to ANCOVAs. Results for the Competence Scale revealed only a main effect of source, $F(1,480)=4.13, p<.05$. When the author was associated with NASA, he or she received higher ratings than when the author was associated with the generic site (NASA $M=6.40$, Spacescience $M=6.17$ ).

Analysis of the objectivity item showed main effects for science involvement, source, and a source by domain interaction. Individuals low as compared to high in involvement considered the source to be more objective (lowinvolvement $M=5.63$, high-involvement $M=5.10$ ). In addition, the author 
TABLE 5

Ratings of the Degree Article Author Is "Objective" as a Function of Source and Domain

\begin{tabular}{lll}
\hline \hline Source & gov &. com \\
\hline NASA & $5.44_{\mathrm{a}}$ & $5.31_{\mathrm{a}, \mathrm{b}}$ \\
Spacescience & $5.87_{\mathrm{a}}$ & $4.73_{\mathrm{b}}$ \\
\hline
\end{tabular}

NOTE: Row and column means with different subscripts differ significantly $(p<.05)$.

was seen as more objective when writing for a .gov site $(M=5.65)$ than for a .com site $(M=5.04)$. The interaction, presented in Table 5, suggests that when the source of the article is NASA, domain has little effect on perceived objectivity. However, when the source is unrecognized, the domain significantly affects perceived objectivity, such that the .gov site author is rated as higher in objectivity than the .com site author.

\section{Discussion: Part 2}

Using a randomized experiment, involving assessment of an actual science article, we found strong support for two of our four hypotheses. We replicated the finding from part 1 that a .gov site is viewed as more credible for science information than a .com site. Respondents considered the source of a science article to have more expertise when the source carried a .gov domain than when it carried a .com domain. In addition, the author of an article appearing at a .gov as compared to a .com domain was viewed as more objective. As predicted by hypothesis 3 , respondents attributed greater credibility to a well-known science name (NASA) than to a generic science name (Spacescience), and this credibility is manifested in several important ways. Most obviously, NASA is seen as more expert (although not more attractive or trustworthy) than Spacescience. In addition, an author who is identified as writing for NASA is viewed as more competent than an author writing for the generic site. Finally, identical articles were judged to be of superior quality if they were described as having appeared at NASA's Web site than if they were described as having appeared at the generic site. No support was found for hypotheses 2 and 4, which predicted that the domain and source effects would be qualified by science involvement. Instead, low- and highinvolvement respondents were equally influenced by source and domain cues.

There are many limitations inherent in studies such as the one presented here, including threats to external validity posed by convenience samples and a restricted sample of experimental stimuli. However, one concern that is a 
somewhat unique aspect of our study results from our decision to administer sequential parts of the study (survey and experiment) in a short time frame to the same participants. In a nutshell, there is a strong likelihood that responses to questions from part 1 (particularly about domain credibility) might have influenced credibility ratings in part 2. Having rated .gov sites as more credible than .com sites in part 1, perhaps participants were especially likely to judge a story from a .gov site as more credible than a .com in part 2. It is not possible to rule out this threat because all participants completed questions from part 1 before completing the experiment in part 2. However, the strength of the domain credibility findings from both parts and the convergence of several different methods for measuring domain credibility provide some assurance that our results are quite robust.

\section{General Discussion}

\section{Implications}

Based on the findings of this study, it appears, as other studies have shown, that a gov site carries more weight in terms of information credibility than does a .com site. In addition, while not a significant relationship, the pattern of means in part 1 suggests that domain may be a more important consideration for low-involvement readers. Put another way, high-involvement readers-those more involved in science and those most likely to obtain science Web information more frequently — may rely less on domain than other credibility cues. These readers judge the quality of the science writing by analyzing the messages carefully. Attracting such readers requires that a site offer high-quality, current, and informative content. However, this should not diminish the importance of domain and site credibility because it is likely that the majority of those surfing the Web for information are low in science involvement. These low-involvement information seekers likely will utilize source credibility cues when choosing a site.

Researchers studying use of the Internet for health information have discovered similar patterns in the ways different groups of health information seekers evaluate health Web sites. Fox and Rainie (2002) characterized about 25 percent of the online health information seekers they surveyed as "vigilant health seekers," another quarter as "concerned health seekers," and the remaining 50 percent as "unconcerned health seekers." Vigilant and concerned health seekers were far more likely than unconcerned health seekers to have a chronic illness; thus, it seems reasonable to suggest that the vigilant and concerned health seekers would be higher than unconcerned seekers in 
involvement with health issues. Fox and Rainie found that vigilant and concerned seekers were more likely to check the sources and posting dates of online health information, more likely to have rejected a health site because they could not determine its source or author, and more likely to discuss the online health content with a health professional. As with our high-involvement science information users, the higher involvement health information seekers also spent more time reading health information online and visited more sites. Thus, for at least these two types of online information, more highly involved users seem to be more likely to use evaluation strategies that focus on the message content or message-related cues, such as its currency.

On the other hand, it is important to acknowledge that our low- and highinvolvement respondents' credibility judgments were influenced equally by source and domain cues; although there were differences in the mean patterns, those differences were not statistically significant. One explanation for this finding is suggested by Rieh and Belkin's (1998) qualitative study of scholars' evaluations of online information. Among this group of respondents - all of whom could be regarded as highly involved - source characteristics, including domain and the name of the sponsoring institution, were key factors in the respondents' judgments about the quality (or credibility) of the information. Educational and government institutions were believed to be most likely to provide credible information because they were perceived to have the most to lose if they did not. Content also was an important characteristic, but it was most important when the information was perceived as useful for them. Fewer respondents mentioned accuracy, which normally would be regarded as a message characteristic of great interest to the highly involved.

Rieh and Belkin's (1998) findings, as well as our own, suggest that Internet users currently may make credibility judgments somewhat differently than users of other media channels. Because obtaining information from the Internet is still relatively new to most people — and perhaps because many Internet users recognize the wide variations in the quality of information that may be available online-characteristics that ordinarily would be regarded as peripheral cues may play a much more important role than they would in other media. In fact, Rieh and Belkin reported that "when respondents mentioned the aspect of source as a criterion of information quality, they immediately referred to the credibility and truthfulness of the source. These characteristics were indicated by the perceived authority of the source" (p. 286). This finding suggests that for this highly involved group, at least, source credibility may be nearly synonymous with message credibility.

Another finding from this study suggests that higher levels of task involvement may lead to greater emphasis on domain and source, again supporting the notion that these characteristics are not regarded as "peripheral" cues for 
online information. Rieh and Belkin's (1998) respondents reported that they are especially cautious about the quality (and therefore the source) of information when the consequences of having incorrect information are more serious.

It may be that peripheral cues such as domain name and the source of the information will continue to be of greater importance to those low in science involvement. People today may take advantage of the accessibility of Internet information to acquire information about a much broader range of topics than they previously would have investigated. However, it is unlikely that casual seekers of science information will be motivated to spend any significant amount of time evaluating the information they find through careful assessment of the information content. Instead, they likely will continue to judge information credibility based primarily on characteristics such as domain and source familiarity. Those who are more highly involved in science, however, may place less emphasis on domain and source as they become more adept at using other types of characteristics, such as the completeness and verifiability of information. Yet another consideration, which needs to be addressed in a future study, is that perhaps, at this point, the likelihood of people trusting a site may be driven more by how useful the site's information is to readers than by anything related to credibility or accuracy.

The ".com fallout" of 2001-02 may imply that surviving and prospering on the Internet will require strategies aimed at differentiating site content from that available at competing sites. This article has examined two such differentiating strategies, site "brand name" and domain, both of which can serve the important differentiation goal of creating credibility. We also have provided evidence that perceived credibility can attract traffic and influence perceptions of the quality of the writers and stories at a site.

\section{Notes}

1. Flanagin and Metzger (2000) did not explain how they defined "entertainment" information.

2. Factor analysis seemed an appropriate way to simplify our scales for several important reasons. First, the studies from which our measures were obtained did not deal specifically with Web site credibility or with the credibility of science information, or both. Motives for seeking science information on the Internet, for example, may be different from motives for obtaining other kinds of Internet information and from motives for obtaining science information from other media. Second, the measures employed in this research were derived from studies of medium credibility (rather than source or spokesperson credibility, for example, Ohanian 1990). Medium credibility has received relatively little empirical attention in comparison to the better known construct of source credibility. Finally, our study combined items from the mediumcredibility literature that have not been administered together in a single study, thereby creating uncertainty as to how the items would fit together. This factor analysis (and all others except 
where noted) was conducted using principal components extraction followed by varimax rotation of factors with eigenvalues greater than 1.0. Items were assigned to a scale if they had loadings in excess of .40 for one factor and below .30 for all remaining factors (see Tabachnick and Fidell 1989, 640). All scales were created by summing items that were cleanly associated with a single factor, except where noted.

\section{References}

Blumler, J. G. 1979. The role of theory in uses and gratifications studies. Communication Research 6:9-36.

Eveland, W. P., Jr., and S. Dunwoody. 1998. Users and navigation patterns of a science World Wide Web site for the public. Public Understanding of Science 7:285-311.

Finberg, H., M. Stone, and D. Lynch. 2001. Digital journalism credibility study. Available from the Online News Association, Knight Foundation: http://www.journalists.org/Programs/ Study.htm.

Flanagin, A. J., and M. J. Metzger. 2000. Perceptions of Internet credibility. Journalism and Mass Communication Quarterly 77 (3): 515-40.

Fox, A., and L. Rainie. 2002. Vital decisions: How Internet users decide what information to trust when they or their loved ones are sick. Washington, DC: Pew Internet \& American Life Project.

Gunther, A. C. 1988. Extremity of attitude and trust in media. Journalism Quarterly 65 (2): 27987.

Hartz, J., and R. Chappell. 1997. Worlds apart: How the distance between science and journalism threatens America's future. Nashville, TN: First Amendment Center.

Hawkins, D. T. 1999. What is credible information? ONLINE, pp. 86-89. Available from http:// www.onlineinc.com/onlinemag.

Health on the Net Foundation. 2001. HONcode site-checker. Available from http://www.hon.ch/ HONcode/HONcode_check.html.

Jenkins, E. 1994. Scientific literacy. In The international encyclopedia of education, vol. 9, 2d ed., edited by T. Husen and T. Postlethwaite, 5345-50. Oxford, UK: Pergamon.

Johnson, H., and J. Scileppi. 1969. Effects of ego-involvement conditions on attitude change to high and low credibility communicators. Journal of Personality and Social Psychology 13:31-36.

Johnson, T. J., and B. Kaye. 1998. Cruising is believing? Comparing Internet and traditional sources on media credibility measures. Journalism and Mass Communication Quarterly 75 (2): $325-40$.

Katz, E., J. G. Blumler, and M. Gurevitch. 1974. Utilization of mass communication by the individual. In The uses of mass communications: Current perspectives on gratifications research, edited by J. G. Blumler and E. Katz, 19-32. Beverly Hills, CA: Sage.

Lasica, J. D. 2002. Online news on a tightrope. Available from http://www.ojr.org/ojr/business.

Marquart, J., G. J. O' Keefe, and A. C. Gunther. 1995. Believing in biotech: Farmers' perceptions of the credibility of BGH information-sources. Science Communication 16 (4): 388-402.

Miller, J. 1998. The measurement of civic scientific literacy. Public Understanding of Science 7 (3): 203-23.

Newhagen, J., and C. Nass. 1989. Differential criteria for evaluating credibility of newspapers and TV news. Journalism Quarterly 66 (2): 277-84. 
Ohanian, R. 1990. Construction and validation of a scale to measure celebrity endorsers' perceived expertise, trustworthiness, and attractiveness. Journal of Advertising 19:39-52.

Papacharissi, Z., and A. Rubin. 2000. Predictors of Internet use. Journal of Broadcasting and Electronic Media 44 (2): 175-96.

Popular Science. 2001. Popular Science: Best of the Web. Available from http:// www.popsci.com/features/bow00.

Rhine, R., and L. Severance. 1970. Ego-involvement, discrepancy, source credibility, and attitude change. Journal of Personality and Social Psychology 16:175-90.

Rieh, S. Y., and N. J. Belkin. 1998. Understanding judgment of information quality and cognitive authority in the WWW. Proceedings of the 61st Annual Meeting of the American Society for Information Science 35:279-89.

Sapp, G. 1992. Science literacy: A discussion and information based definition. College and Research Libraries, p. 53.

Sternthal, B., L. W. Phillips, and R. Dholakia. 1978. The persuasive effect of source credibility: A situational analysis. Public Opinion Quarterly 42 (3): 285-314.

Sundar, S. S. 1999. Exploring receivers' criteria for perception of print and online news. Journalism and Mass Communication Quarterly 76 (2): 373-86.

Tabachnick, B. G., and L. S. Fidell. 1989. Using multivariate statistics, 2d ed. New York: Harper and Row.

Weigold, M. F. 2002. Communicating science: A review of the literature. Science Communication 23 (2): 164-93.

Wiener, J. L., and J. C. Mowen. 1986. Source credibility: On the independent effects of trust and expertise. In Advances in consumer research, vol. XIII, edited by R. J. Lutz, 306-10. Provo, UT: Association for Consumer Research.

DEBBIE TREISE is a professor of advertising at the University of Florida. She received her Ph.D. from the University of Tennessee in communications in 1992. She teaches graduate research and science/health seminars. Her research interests are science and health communications.

KIM WALSH-CHILDERS is an associate professor in journalism at the University of Florida. She received her Ph.D. in 1990. She teaches feature writing, journalism, and media ethics. Her research focuses on print media news coverage of health issues, mass media effects on individual health, and the relationship between mass media content and adolescent sexual beliefs and behavior.

MICHAEL F. WEIGOLD is an associate professor at the University of Florida. He teaches advertising and graduate courses in mass communications. He earned his Ph.D. in social psychology in 1989. His current research interests are in science and health communication.

MEREDITH FRIEDMAN is a master's degree graduate from the University of Florida. She currently works for a product placement firm in San Francisco. 